\title{
Effects of Exercise on the Therapeutic and Rehabilitation Process of Patients with Multiple Sclerosis- A Narrative Review
}

\author{
Payam Saadat ${ }^{1}$, Seyed Mohammad Masood Hojjati², Hoda Naghshineh ${ }^{3}$, Alijan Ahmadi Ahangar ${ }^{4}$, Ali Alizadeh Khatir ${ }^{5}$ \\ ${ }^{1}$ Associate Professor of Neurology, Mobility Impairment Research Center, Health Research Institute, Babol University \\ of Medical Sciences, Babol, Iran. ${ }^{2}$ Assistant Professor of Neurology, Mobility Impairment Research Center, Health \\ Research Institute, Babol University of Medical Sciences, Babol, Iran. ${ }^{3}$ Assistant Professor of Neurology, Department of \\ Neurology, Babol University of Medical Sciences, Babol, Iran. ${ }^{4}$ Professor of Neurology, Mobility Impairment Research \\ Center, Health Research Institute, Babol University of Medical Sciences, Babol, Iran. ${ }^{5}$ Assistant Professor of Neurology, \\ Mobility Impairment Research Center, Health Research Institute, \\ Babol University of Medical Sciences, Babol, Iran.
}

\section{ABSTRACT}

\section{BACKGROUND}

Multiple sclerosis (MS) is a chronic, autoimmune and inflammatory nervous system disease. It leads to the loss of myelin in the white matter of brain, spinal cord and optic nerves, and causes tiredness, muscle cramp, tremor, unsteady gait and some inability to move. Due to the effects of exercise in patients with MS, we aimed to evaluate the effect of exercise on the therapeutic and recovery process of patients with MS. In this study, a narrative review was conducted using keywords, including Multiple Sclerosis, exercise, recovery, treatment, through searching in international scientific databases, such as PubMed, Web of Science and Scopus, on articles published form inception. Different types of exercises were assessed and reported by the researchers as beneficial rehabilitation strategies for patients to manage symptoms restore function, optimize quality of life, promote wellness and back to activities of daily living. These exercises include endurance exercises (aerobics), balance exercises, flexibility exercises, yoga exercises, hypnosis exercises, physiotherapy exercises and exercise in water. Because of positive effects of sport exercises, it is recommended that training program be considered as a complementary treatment alongside other treatments for MS patients.

\section{KEY WORDS}

Multiple Sclerosis, Exercise, Rehabilitation, Therapy
Corresponding Author: Dr. Seyed Mohammad Massod Hojjati, Department of Neurology, Babol University of Medical Sciences, Ganjafrooz Street, Babol, Mazandaran, Iran.

E-mail:

hojjatiseyedmohammadmasood@gmail.com DOI: $10.14260 /$ jemds/2019/635

Financial or Other Competing Interests: None.

How to Cite This Article:

Saadat P, Hojjati SMM, Naghshineh H, et al. Effects of exercise on the therapeutic and rehabilitation process of patients with multiple sclerosis- a narrative review. J. Evolution Med. Dent. Sci. 2019;8(38): 2924-2928, DOI: 10.14260/jemds/2019/635

Submission 17-07-2019, Peer Review 08-09-2019, Acceptance 14-09-2019, Published 23-09-2019. 


\section{BACKGROUND}

Multiple Sclerosis (MS) is one of the most common inflammatory Central Nerve System (CNS) diseases that affect a wide range of neurological functions.(1) This disease is caused by damage to the myelin sheath.(2) Patients with this disease are faced with a wide range of problems, from a simple sensory problem to paralysis of all four organs. In addition, due to the chronicity of the disease and frequent attacks, it often leads to sensitiveness and suffering, fatigue, depression and decreased self-confidence in patients. (3) Unfortunately, many people in the world are suffering from this disease and their number is increasing day by day. (4) This disease affects more than 2.5 million people in the world, mostly between second and fourth decades of life.(5,6) The sense of fatigue in patients with MS is often mild and sever at the onset of the disease, which will be with the patient during the disease course.(7) Fatigue can also occur in the form of symptoms such as unbearable lethargy, early fatigue, and energy depletion in the patient. Despite the unknown mechanism of its occurrence, neurohormonal factors and autoimmune mechanisms are among the most important factors in the development of fatigue in these patients. $\left.{ }^{8}\right)$ Given the ever-increasing rate of mortality among people suffering from this disease, high costs and drug-related complications and problems, it seems reasonable to use nonprescriptive methods that can reduce problems and improve the function of patients with MS.(9) In addition to these complications, this disease generally causes a tendency to inactivity in the person that can cause other secondary diseases in the patient. On the other hand, exercise can slow down its development and can partially disrupt the progress of disease by creating protective features and possibly nerve rehabilitation. However, there are still disagreements about the types of exercise.

Many studies have shown that various interventions can improve cramps in these patients, which include exercises using balance sheet, whole body shaking, hypnotherapy, physiotherapy based on the concept of boost, motor exercises, water therapy, progressive resistance exercises, and aerobic exercises. Over the past few years, a particular attention has been paid to exercise and the importance of aerobic power, especially aerobic exercise.(10) Examining the relationship between physical activities and life quality of patients with MS showed that patients with higher levels of physical activity have a better life quality than other patients, and they experience less fatigue, and exercise allows each of the various muscle parts to interact with each other. In each joint, there is a desired range of motion that is essential for maximum performance. Flexibility is important not only for doing physical activities, but also for preventing damage. Lack of activity causes the joints to lose their flexibility, because connective tissues become shorter.(11) In another study, Kargarfard et al. showed that 8-week regular water exercises in patients with MS have improved their fatigue and life quality.(12) A number of conducted studies indicate positive effects of physical activity on aerobic power and life quality of people.(13) While some other studies have not shown such results.(14)

The role of exercise in patients with MS has been controversial and somebody think that this can lead to an increased injury of the disease. However, recent research shows the benefits of exercise regardless of the level of secondary disability of patients with MS. Considering the necessity of reviewing the improving factors of patients with MS by exercise and recognizing the recovery or therapeutic strategy, the present study was conducted to review the effect of exercise on the therapeutic and recovery process of patients with MS.

\section{Search Strategy and Results}

In this study, a narrative review was conducted using keywords, including Multiple Sclerosis (MS), exercise, recovery, treatment, through searching in international scientific databases, such as PubMed, Web of Science and Scopus, on articles published form inception. Irrelevant sources and articles were excluded. Assessing the papers showed that many measures have been taken to reduce the problems and improve the level of daily living activities in patients with MS, however, each has had side effects. In recent years, non-pharmacological approaches have attracted patients, including patients with MS. Exercise is an example of these therapies. Comprehensive therapies are used to increase the physical and mental comfort of patients.(15)

We have summarized the different exercises and activities affecting improvement of the patients with MS as follows-

\section{Endurance Exercises (Aerobic)}

In patients with MS, the knowledge of aerobic power can be effective in preparing a plan for regular exercises and increasing physical activities. Kicking the fixed bike, walking, and low intensity aerobic exercises on the chair are good choices that are chosen according to the person's personal interest and the type and amount of his physical problems. Endurance exercises are a kind of exercise that make muscles resist against a type of pressure or damage, such as lifting weight or using exercise groups. There are various types of resistance training, but most of them are aimed to increase muscles' power and performance. It was advised to the patients with MS to avoid exercise (Endurance and resistance) with the fear of disease progression. However, over the past 10 to 15 years, several studies examined the effects of resistance training on patients with MS. These studies show that there are promising results for improving the life quality of patients with MS.(16-18)

\section{Balance Exercises}

The effects of the endurance exercises are not just on the mobility and power, but are directly on the balance. Expansion muscle power is for leg strength, and for balance, there are exercises related to leg activities, including: getting up from the chair, sudden jump, getting up on the heels and twisting the foot, and combined exercises will affect their balance and performance.(19)

\section{Flexibility Exercises}

It is advisable to maximize the time of stretching exercises in these patients from 30 to 60 seconds and repeat them 3 to 5 times. However, sometimes doing stretching exercises in patients with muscular cramps needs more time. In such a situation, a stretching exercise should be done for 10 to 20 minutes to one hour but with low intensity. $(19,20)$ 


\section{Yoga Exercises}

Muscle weakness is one of the most common symptoms of MS disease, and is usually referred to as early symptoms. At least, half of the affected people experience muscle fatigue that is a "fatigue phenomenon". The effects of Yoga and aerobic exercise on cognitive function, fatigue, mood, and life quality on patients with MS were reviewed. Then, it was found that people with MS who participated in a 6-month Yoga or exercise class, had significantly improved fatigue compared to the control group. ${ }^{(21,22)}$ Moreover, in this regard, a study conducted on women with MS showed that Yoga can recover inflammation that indicates a reduction in Cortisol level and an increase in adrenocorticotropic hormone ACTH level.(23)

\section{Hypnosis Exercises}

Various reports show that hypnosis can affect different physical damages. Several studies show that hypnosis can be useful in the treatment of physical disorder caused by MS. $(24,25)$

\section{Physiotherapy Exercises}

Currently, there are several randomized controlled experiments in rehabilitation packages, physiotherapy, or specific and non-specific techniques with MS.(26) Generally, this study and review studies report the benefits of these patients' recovery. Nevertheless, some problems may also raise in some studies. Our study found that physiotherapy methods are effective for the recovery of patients with MS. A combination of appropriate physiotherapy techniques, and appropriate to the progression of the disease in patients with MS is effective in multiple rehabilitation. $(22,27,28)$

\section{Exercise in Water}

Using water therapy (exercise in water) has been tested as a therapeutic factor for MS. Exercise in water creates a floating and safe environment for these patients. Warm water at 80 to 84 Fahrenheit degree $\left(30\right.$ to $31^{\circ} \mathrm{C}$ ) reduces muscle stiffness and allows the patient to move more easily. The best exercise for old-age patients with leg sensory defects is aerobic exercise in water. Although exercise in water does not return what is destroyed and lost, due to the therapeutic properties of water, it improves endurance and coordination of the patient.(29) Three main advantages of exercise in water are: flotation, adhesion, and thermodynamics. Flotation is useful in exercise in water, because reduces the weight pressure on joints, and creates a very smooth practice environment. It creates stretch viscosity, and is an environment in which exercises can be done in all directions.(30)

\section{DISCUSSION}

MS is a chronic and debilitating disease of the nervous system, which is specified by inflammation at any point of the spinal cord and the brain.(26) The prevalence of this disease in women is two times more than men.(31) Patients with MS fear of activity. Lack of balance in walking, tremor, instability while walking, dizziness, sudden movement of organs, body inconsistency and weakness prevent these patients from taking part in the social arena.(3) There are several studies that highlight the benefits of exercise in the regulation of MS disease. Many of them have limitations on the number of participants, mental scoring systems, and study duration. As this field has evolved, particularly over the last decade, different articles have been published that show the benefits of exercise in patients with MS. In a study conducted by Rampello et al, the effect of 8-week aerobic exercise on the MS disease was examined and it was found that aerobic exercises significantly increase the walking speed and distance of patients with MS.(32) In short, it can be stated that exercise can improve the functional endurance in the combined exercise group. Some improvement was also observed in fatigue and life quality. It can be concluded that combined exercises have significantly improved physical fitness (Functional endurance) in the patients with MS. By enhancing the functional endurance through combined exercises, they can help them facilitating doing their daily work at home and office. It also presented a solution to prevent their weakness and loss of healthy and young force in the community. Motl et al. studied the effect of combined improving exercise on patients with significant inability in MS. Combined exercises could significantly improve walking, walking speed and mobility of patients.(33) Patients who participate in exercises programs have more physical and cardiovascular fitness level than others, and feel less fatigue, and have a positive attitude and more participation in social activities. Therefore, it can be stated that exercise plays an important role in the treatment, recovery, capacity maintenance, function and ability of patients with MS.

Exercise is the low-cost form of treatment that has been recognized as the positive and real way for patients with MS. Participation in the exercise program has the potential for the recovery of many victims of this disease. Research can determine the amount of progress achieved through physical training. In addition, physical therapy and exercise have a positive effect on behaviour and trust in most patients, as much as they suffer from a physical illness, mental state can lead to paralysis and reduced function of patients.(34) Generally, according to the current research, it can clearly be concluded that exercise can be the basis of treating patients with MS. It has been shown that any of the mentioned exercises can have a positive effect on all types of symptoms commonly seen in patients with MS.(35) Exercise leads to a significant improvement in life quality without any negative impact, because all studies that track adverse results didn't show an increase in the exacerbation of the disease or events. Note that it doesn't seem that exercise prevent or reduce the frequency of recurrence of the disease, but it improves endurance and coordination of the patient.(36) In the studies, there was an attempt to assess whether there is a difference between aerobic exercise and the combined program of aerobic exercises with resistance exercises on the impact of life quality and fatigue.(37) In these studies, it was also shown that any group of patients with MS who performed exercise, had a significant difference from the control group.

As mentioned above, water exercises have an evolutionary background with a high potential in patients with MS.(38) These types of exercises are appropriate for patients, because in the water, body temperature is lower than similar exercise in non-water environment. As a result, patients with MS are less likely to stop exercise, because they are afraid of symptoms of increased body temperature.(29) Accordingly, therapeutic exercises as a non-invasive treatment, are an effective factor in improving the ability to 
perform daily activities, and can be recommended along with taking medication to patients with MS. Therefore, this exercise can be recommended to patients with MS, physiotherapists, doctors, sports instructors, and all people who are somehow trying to treat and recover these patients.(39) Findings show that aerobic exercises and physiotherapy improve MS patients' satisfaction with their physical, psychological, and social performance. It may also be considered as a natural way to treat MS disease.(40)

In this study, the results of various studies in relation to the effect of non-pharmacological method, i.e. exercise, on the fatigue of patients with MS were used to be compared with the results of the present study. However, there was lack of a study that can take into account the patient and his family. In fact, the basic benefits of regular exercises including increased cardio-respiratory fitness, increased muscular strength and endurance, reduced fatigue, improved morale, and increased ability to do daily work with more power. These benefits are significantly effective in controlling the symptoms of MS disease and improving health. Moreover, since these patients repeatedly experience weakness in their lower extremity and fatigue, and on the other hand, as resistance exercises using rubber band were focused on the lower extremities of these patients, as a result, patients may overcome the weakness of their lower extremities that often develop as fatigue and disturbed walking, and subsequently, these exercises significantly reduce fatigue after exercises in MS patients. In this regard, resistance exercises with rubber band have often shown better results than other traditional exercises. ${ }^{(41)}$

\section{CONCLUSIONS}

In a comprehensive review and evaluation of several methods to deal with fatigue, Rutolli et al.(42) concluded that exercise plays an important role in preventing and treating fatigue in MS patients. In their review, they stated that 80 percent of all MS patients were suffering from disability, and 55 percent of patients said that they were suffering from unpleasant symptoms. In MS patients and other local diseases that have no definitive treatment, the main goal of treatment and scientific studies is to improve the quality of life of these individuals. The conducted studies paid attention to a combination of these exercises considering the significance of water and aerobic exercises in these patients and attempted to compensate the defects of past exercises. Due to the importance of the mentioned benefits, doing these exercises is recommended as an effective and efficient way for MS patients' treatment. Note that the fatigue amount used in their reviews, varies widely in different studies. However, they concluded that the best treatment for fatigue in MS patients is exercise as a part of a multidisciplinary approach. Although exercise does not return what is lost, due to the therapeutic characteristics examined in this study, it improves both endurance power and coordination of the patient. However, all these findings indicate that exercise can result in patients' recovery.

\section{REFERENCES}

[1] Prasad KN, Mishra AM, Gupta D, et al. Analysis of microbial etiology and mortality in patients with brain abscess. J Infect 2006;53(4):221-7.

[2] Hojjati SM, Zarghami A, Hojjati SA, et al. Optic neuritis, the most common initial presenting manifestation of multiple sclerosis in northern Iran. Caspian J Intern Med 2015;6(3):151-5.

[3] Hojati S, Zarghami A, Yousefzad T, et al. Epidemiological features of 263 patients with multiple sclerosis residing in Babol, Iran. J Babol Univ Med Sci 2016;18(1):52-6.

[4] Rahn EJ, Iannitti T, Donahue RR, et al. Sex differences in a mouse model of multiple sclerosis: neuropathic pain behavior in females but not males and protection from neurological deficits during proestrus. J Biology of Sex Differences 2014;5(1):4.

[5] Marrie RA, Horwitz RI. Emerging effects of comorbidities on multiple sclerosis. The Lancet Neurology 2010;9(8):820-8.

[6] Hojjati SMM, Hojjati SA, Baes M, et al. Relation between EDSS and monosymptomatic or polysymptomatic onset in clinical manifestations of multiple sclerosis in Babol, northern Iran. Caspian J Intern Med 2014;5(1):5-8.

[7] Matuska K, Mathiowetz V, Finlayson M. Use and perceived effectiveness of energy conservation strategies for managing multiple sclerosis fatigue. Am J Occupational Therapy 2007;61(1):62-9.

[8] Masoudi R, Mohamady E, Nabavi M, et al. Effect of Orem's self-care theory on the quality of life in multiple sclerosis pations. Shahrkord Univ of Med Sci 2008;10(2):21-9. [Persian]

[9] Shafizadeh M, Platt GK, Mohammadi B. Effects of different focus of attention rehabilitative training on gait performance in Multiple Sclerosis patients. J Bodywork and Movement Therapies 2013;17(1):2834.

[10] Eftekhari E, Nikbakht HA, Rabiei K, et al. Effect of endurance training on aerobic power and quality of life in female patients with multiple sclerosis. Olympic Spring 2008;16(1):37-46.

[11] Motl RW, McAuley E, Snook EM, et al. Physical activity and quality of life in multiple sclerosis: intermediary roles of disability, fatigue, mood, pain, self-efficacy and social support. Psychology, Health \& Medicine 2009;14(1):111-24.

[12] Kargarfard M, Etemadifar M, Asfarjani F, et al. Changes in quality of life and fatigue in women with multiple sclerosis after 8 weeks of aquatic exercise training. J Fundamentals of Mental Health 2010;12(3):562-73.

[13] Sakkas GK, Hadjigeorgiou GM, Karatzaferi C, et al. Intradialytic aerobic exercise training ameliorates symptoms of restless legs syndrome and improves functional capacity in patients on hemodialysis: a pilot study. ASAIO Journal 2008;54(2):185-90.

[14] Ohayon MM, Roth T. Prevalence of restless legs syndrome and periodic limb movement disorder in the general population. J Psychosomatic Research 2002;53(1):547-54. 
[15] Mount J, Dacko S. Effects of dorsiflexor endurance exercises on foot drop secondary to multiple sclerosis: a pilot study. NeuroRehabilitation 2006;21(1):43-50.

[16] Taylor NF, Dodd KJ, Prasad D, et al. Progressive resistance exercise for people with multiple sclerosis. Disability and Rehabilitation 2006;28(18):1119-26.

[17] Petajan JH, Gappmaier E, White AT, et al. Impact of aerobic training on fitness and quality of life in multiple sclerosis. Annals of Neurology 1996;39(4):432-41.

[18] Mostert S, Kesselring J. Effects of a short-term exercise training program on aerobic fitness, fatigue, health perception and activity level of subjects with multiple sclerosis. J Multiple Sclerosis 2002;8(2):161-8.

[19] Monireh MN, Hossein S, Fatemeh H. Effects of selected combined training on balance and functional capacity in women with multiple sclerosis. World Appl Sci J 2012;16(7):1019-26.

[20] Golzari Z, Shabkhiz F, Soudi S, et al. Combined exercise training reduces IFN- $\gamma$ and IL-17 levels in the plasma and the supernatant of peripheral blood mononuclear cells in women with multiple sclerosis. International immunopharmacology 2010;10(11):1415-9.

[21] Oken BS, Kishiyama S, Zajdel D, et al. Randomized controlled trial of yoga and exercise in multiple sclerosis. Neurology 2004;62(11):2058-64.

[22] Alphonsus KB, Su Y, D'Arcy C. The effect of exercise, yoga and physiotherapy on the quality of life of people with multiple sclerosis: systematic review and metaanalysis. Complementary Therapies in Medicine 2019;43:188-95.

[23] Najafi P, Moghadasi M. The effect of yoga training on enhancement of Adrenocorticotropic hormone (ACTH) and cortisol levels in female patients with multiple sclerosis. Complementary Therapies in Clinical Practice 2017;26:21-5.

[24] Sutcher H. Hypnosis as adjunctive therapy for multiple sclerosis: a progress report. American J Clinical Hypnosis 1997;39(4):283-90.

[25] Braley TJ, Segal BM, Chervin RD. Hypnotic use and fatigue in multiple sclerosis. Sleep Medicine 2015;16(1):131-7.

[26] Jailkhani SMK, Naik JD, Thakur MS, et al. Retrospective analysis of poisoning cases admitted in a tertiary care hospital. Int J Recent Trends Sci Technol 2014;10(2):365-8.

[27] Armutlu K, Karabudak R, Nurlu G. Physiotherapy approaches in the treatment of ataxic multiple sclerosis: a pilot study. Neurorehabilitation and Neural Repair 2001;15(3):203-11.

[28] Wiles CM. Physiotherapy and related activities in multiple sclerosis. J Multiple Sclerosis 2008;14(7):863-71.

[29] Shanazari Z, Moradi MR, Mehrabi HA. Effect of aquatic training on psychological well-being in women with multiple sclerosis. Knowledge \& Health 2015;10(1):19-24.
[30] Frohman AN, Okuda DT, Beh S, et al. Aquatic training in MS: neurotherapeutic impact upon quality of life. Annals of Clinical and Translational Neurology 2015;2(8):864-72.

[31] Krupp LB, LaRocca NG, Muir-Nash J, et al. The fatigue severity scale. Application to patients with multiple sclerosis and systemic lupus erythematosus. Archives of Neurology 1989;46(10):1121-3.

[32] Rampello A, Franceschini M, Piepoli M, et al. Effect of aerobic training on walking capacity and maximal exercise tolerance in patients with multiple sclerosis: a randomized crossover controlled study. Physical Therapy 2007;87(5):545-55.

[33] Motl RW, Smith DC, Elliott J, et al. Combined training improves walking mobility in persons with significant disability from multiple sclerosis: a pilot study. J Neurologic Physical Therapy 2012;36(1):32-7.

[34] Aidar FJ, De Matos GD, De Souza RF, et al. Influence of aquatic exercises in physical condition in patients with multiple sclerosis. J Sports Medicine and Physical Fitness 2018;58(5):684-9.

[35] Cortese M, Riise T, Bjørnevik K, et al. Body size and physical exercise and the risk of multiple sclerosis. Multiple Sclerosis Journal 2018;24(3):270-8.

[36] Kinnett-Hopkins D, Learmonth Y, Hubbard E, et al. The interpretation of physical activity, exercise and sedentary behaviours by persons with multiple sclerosis. Disability and Rehabilitation 2019;41(2):166-71.

[37] Felippe LA, Salgado PR, de Souza SD, et al. A controlled clinical trial on the effects of exercise on cognition and mobility in adults with multiple sclerosis. Am J Physical Medicine \& Rehabilitation 2019;98(2):97102.

[38] Stennett A, De Souza L, Norris M. Physical activity and exercise priorities in community dwelling people with multiple sclerosis: a Delphi study. Disability and Rehabilitation 2018;40(14):1686-93.

[39] Motl RW, Pekmezi D, Wingo BC. Promotion of physical activity and exercise in multiple sclerosis: Importance of behavioral science and theory. J Multiple Sclerosis Experimental, Translational and Clinical 2018;4(3):2055217318786745.

[40] Sangelaji B, Kordi M, Banihashemi F, et al. A combined exercise model for improving muscle strength, balance, walking distance and motor agility in multiple sclerosis patients: a randomized clinical trial. Iranian J Neurology 2016;15(3):111-20.

[41] Ghigiarelli JJ, Nagle EF, Gross FL, et al. The effects of a 7-week heavy elastic band and weight chain program on upper-body strength and upper-body power in a sample of division 1-AA football players. J Strength \& Conditioning Research 2009;23(3):756-64.

[42] Rottoli M, La Gioia S, Frigeni B, et al. Pathophysiology, assessment and management of multiple sclerosis fatigue: an update. Expert Review of Neurotherapeutics 2017;17(4):373-9. 